\title{
Fluoxetine in the Treatment of Premenstrual Dysphoria
}

Tung-Ping Su, M.D., Peter J. Schmidt, M.D., Merry A. Danaceau, R.N., M.S.N., Marie B. Tobin, M.D., Donald L. Rosenstein, M.D., Dennis L. Murphy, M.D., and David R. Rubinow, M.D.

We performed a double-blind, placebo-controlled, crossover trial of fluoxetine in 17 women with prospectively confirmed PMS who also met criteria for premenstrual dysphoric disorder (PMDD). A subset of 10 women with $P M D D$ and an additional 10 controls participated in a single-dose $m$-chlorophenylpiperazine ( $m$-CPP) challenge during the follicular and luteal phases of the menstrual cycle. We evaluated the ability of the acute behavioral response to luteal phase $m-C P P$ administration to predict therapeutic response to fluoxetine. Compared with baseline, fluoxetine, but not placebo, treatment significantly improved both emotional and physical symptoms. We identified 11 (65\%) fluoxetine responders who no longer met diagnostic criteria for PMDD during fluoxetine but remained symptomatic during placebo treatment. In addition, acute symptomatic improvement also occurred following $m$-CPP administration in 7 of 10 women with $P M D D$. The small number of $m-C P P$ nonresponders did not respond to fluoxetine either. Our findings confirm that fluoxetine is an effective treatment of PMDD.

[Neuropsychopharmacology 16:346-356, 1997]

(C) Published 1997 by Elsevier Science Inc.
KEY WORDS: Fluoxetine; Serotonin; Premenstrual dysphoric disorder (PMDD); $m$-Chlorophenylpiperazine ( $m$-CPP); Follicular phase; Luteal phase

Premenstrual syndrome (PMS) describes a heterogenous group of affective and somatic symptoms that occur in a phase-specific manner during the luteal phase of the menstrual cycle. Investigators have identified a role of serotonin (5-HT) function in many of the behaviors commonly experienced by women with PMS, including alterations in mood, appetite, sleep, sexual interest, and impulsivity (Spoont 1992). In addition, although therapeutic inconsistencies with some psychotropic agents have been demonstrated (Smith et al. 1987; Harrison et al. 1990; Schmidt et al. 1993), serotonergic pharmacological agents [e.g., clomipramine (Eriksson et al. 1990;

From the National Institute of Mental Health, Bethesda, Maryland. Address correspondence to: Tung-Ping Su, M.D., National Institute of Mental Health, Building 10, Room 3N238, 10 Center Drive, MSC 1276, Bethesda, MD 20892-1276.

Received April 8, 1996; revised September 24, 1996; accepted October 17, 1996.
Sundblad et al. 1992, 1993), buspirone (Rickels et al. 1989) and fluoxetine (Stone et al. 1991; Menkes et al. 1992; Wood et al. 1992)] appear superior to placebo in alleviating PMS symptoms, suggesting a possible role of serotonin system dysregulation in PMS. Further, pharmacological challenge studies, using either the serotonin precursor tryptophan or the serotonin agonist m-chlorophenylpiperazine (m-CPP), have been reported to stimulate differential neuroendocrine response patterns in women in PMS compared with controls (Halbreich 1990; Bancroft et al. 1991).

Two double-blind, placebo-controlled, crossover (Wood et al. 1992; Menkes et al. 1993) and two parallel (Stone et al. 1991; Steiner et al. 1995) design studies have reported the therapeutic efficacy of fluoxetine (dose range 20 to $60 \mathrm{mg}$ ) in women with prospectively confirmed diagnoses of PMS. Despite evidence that on a group basis fluoxetine is superior to placebo in treating PMS, all four studies identified some subjects who demonstrated a partial or nonresponse to fluoxetine, suggesting that treatment response to fluoxetine may vary even in women with prospectively confirmed PMS. More- 
over, three studies (Stone et al. 1991; Menkes et al. 1992; Steiner et al. 1995) reported that remission of PMS occurred in approximately $50 \%$ of subjects treated. Investigators have suggested a number of possible explanations for the inconsistent response to fluoxetine in PMS, including individual differences in dosage requirements and the presence of a past psychiatric history. Similarly, variable response to fluoxetine has been reported in other conditions (Hollander et al. 1993), and one study has suggested that nonresponse to serotonergic agents such as fluoxetine or clomipramine in obsessive-compulsive disorder can be predicted by the behavioral and neuroendocrine response to the 5-HT probe m-CPP (Hollander et al. 1993). In this study we attempted to replicate and extend previous investigations of the therapeutic effects of fluoxetine in women with prospectively confirmed PMS. In a published, relatively large, parallel design study of fluoxetine in PMS, a moderate therapeutic response was observed in 53\% of fluoxetine-treated cycles and $28 \%$ of placebo-treated cycles (Steiner et al. 1995). What remains unresolved is the question of the number of patients who will respond to active medication, but not placebo, a question that is of critical clinical importance, given the high placebo response rate and the high intersubject variability in the presenting symptoms of women with PMS. We have addressed this question with a double-blind, placebo-controlled crossover design. In addition, in this study, we evaluated the ability of the behavioral response to a serotonin agonist, $\mathrm{m}-\mathrm{CPP}$, administered at baseline prior to study entry, to predict the therapeutic response to fluoxetine in women with PMS.

\section{SUBJECTS AND METHODS}

\section{Subject Selection}

The study participants $(n=19)$ were either self-referred in response to advertisements in the local newspapers and hospital newsletter or were referred by their physicians. All women were screened for the absence of significant medical illness (current or in the past year) through history, physical examination, and routine laboratory tests. A structured psychiatric interview, the Structured Clinical Interview for DSM-III-R (SCID) (Spitzer et al. 1989), was administered to each subject to confirm the absence of significant current or recent (past 2 years) Axis I psychiatric illness, including alcohol and substance abuse. A past history of Axis I psychiatric illness (more than 2 years prior to study entry) was not an exclusion criterion for the women with PMS in contrast to controls for the m-CPP study (see later). The presence of Axis II psychiatric diagnoses was not determined. Except for one woman who was on a stable regimen of thyroid hormone replacement for hypothyroidism, none of the women had been taking psychoac- tive medications, hormonal preparations (including oral contraceptives), mineral supplements, or nonsteroidal anti-inflammatory medications within the past 6 months. All women reported having regular menstrual cycles ranging from 23 to 35 days. Before participating in the study, each woman had the diagnosis of PMS prospectively confirmed by daily, three-item, visual analog scale self-ratings for 3 months. Each participant had at least a $30 \%$ increase in mean negative mood (e.g., depression, anxiety, irritability) symptoms, relative to the actual range of the analog scale used, in the week before menses compared with the week after menses in at least two of three cycles. An exclusion criterion was the appearance of significant mood symptoms (mean mood ratings below the midpoint of the rating scale) during the follicular phase of the menstrual cycle. Approximately $30 \%$ of the women presenting to our clinic with symptoms of PMS and the absence of conditions precluding participation in our study met these diagnostic criteria. In addition, we retrospectively examined the records of the women participating in the study and found that all those selected for participation also met the criteria for premenstrual dysphoric disorder (PMDD) of the Diagnostic and Statistical Manual of Mental Disorders, 4 th edition (1994). To emphasize the severity of the affective symptoms experienced by the women in our sample, we refer to them throughout the remainder of this paper as having PMDD. Pregnancy tests were performed before the beginning of the study, and all participants were required to employ barrier contraceptive methods to prevent pregnancy during the course of the study. The protocol was reviewed and approved by the National Institute of Mental Health Intramural Research Review Board, and oral and written informed consents were obtained from all patients before the study started.

Control subjects for the $\mathrm{m}-\mathrm{CPP}$ challenge were women $(n=10)$ who had the absence of significant PMS symptoms confirmed in a manner similar to the patient group and who had no past or current Axis I psychiatric illness.

\section{Study Design}

Therapeutic Trial of Fluoxetine. All patient subjects entered a randomized, double-blind (patient and rater), placebo-controlled, crossover design trial of fluoxetine. Fluoxetine (Eli Lilly, Indianapolis, IN) and placebo were each administered for three consecutive menstrual cycles followed by one washout menstrual cycle (approximately 1 month) to ensure an adequate elimination of fluoxetine and its metabolites. The entire study period was eight menstrual cycles, including six cycles of the trial (double-blind) and two cycles of washout. Medication was administered at a dose of two tablets per day starting on the first day of the menses 
and continuing for a full menstrual cycle. Each tablet contained $10 \mathrm{mg}$ of fluoxetine or placebo. In the next two cycles, the dosing schedule was flexible; the dose could be unchanged or increased by one or two tablets for each cycle as determined by PMDD symptom severity or treatment side effects. The dosage ceiling was six tablets per day (i.e., $60 \mathrm{mg}$ fluoxetine or placebo). Lower doses of fluoxetine or placebo were employed during the study if side effects precluded continuation at the same dose. According to clinical needs, daily dose could be single or divided, and the time of administration was flexible. Medication was tapered by 10 to $20 \mathrm{mg}$ every 2 to 3 days over 1 week at the end of the third treatment cycle after each trial phase (fluoxetine or placebo).

Oral $m$-CPP Challenge Test. Following acceptance into this study, and prior to entering into the therapeutic trial, patients were requested to undergo two oral m$\mathrm{CPP}$ challenge tests during two consecutive menstrual cycle phases: the late luteal ( $2-12$ days prior to menses) and the mid-follicular (3-9 days after the start of menses) phases. Participation in the $\mathrm{m}-\mathrm{CPP}$ challenge was optional, and the order of performance of $\mathrm{m}$-CPP infusions was randomized. In brief, this procedure [detailed elsewhere (Murphy et al. 1989)] consists of oral administration of $0.5 \mathrm{mg} / \mathrm{kg} \mathrm{m}-\mathrm{CPP}$ followed by temperature measurement (every 15 minutes) and plasma sampling as well as visual analog symptom self-ratings (every 30 minutes) for 3.5 hours.

\section{Behavioral Ratings}

Daily Ratings. Patients were required to complete daily self-report symptom ratings during the entire study period, which included three cycles of baseline ratings (the screening phase and the $\mathrm{M}-\mathrm{CPP}$ tests) and the eight cycles of trial ratings (six cycles of fluoxetine/ placebo and two cycles of washout). Daily ratings consisted of a 16-item extended version of the visual analog scale (VAS) and a 21-item six-point scale representing a slight modification of the Daily Rating Form (DRF) (Endicott and Halbreich 1982; Halbreich et al. 1985). Both of the self-rating scales assess the severity of common symptoms of PMDD, including (1) mood symptoms, such as sadness, anxiety, irritability, and mood swings; (2) physical symptoms, such as breast pain and bloating; and (3) other symptoms, such as fatigue, food cravings, impaired social and work functioning, impulsiveness, and global impairment. The DRF ratings also measure disturbances of sleep and sexual interest.

Composite Symptom Ratings. A composite symptom score was calculated for each woman and represented the mean of the daily symptom ratings (DRF, VAS) during the premenstrual and postmenstrual weeks for each month during each drug condition. The symptoms incorporated into the composite scores consisted of composite mood symptoms (irritability, mood lability, sad- ness, and anxiety) and composite social impairment symptoms (social isolation and work inefficiency).

Cross-Sectional Ratings. All women were seen during the follicular and luteal phases of each menstrual cycle throughout this study at the National Institute of Mental Health outpatient clinic. During the visits, each woman completed the following rating scales: Beck Depression Inventory (BDI) (Beck et al. 1961); State Trait Anxiety Inventory-State Form (STAI) developed by Spielberger et al. (1970); the Rating Scale for Premenstrual Tension Syndrome (Steiner et al. 1980), both selfrated (PMTS-Self) and observer-rated (PMTS-Rater) forms; and a physical symptom checklist (VanKammen and Murphy 1975) to detect the side effects of fluoxetine. The raters (T.S. and M.D.) completing the PMTSRater scale were blind to the medication status. During the late luteal phase visit, blood samples were collected to measure plasma progesterone levels, were centrifuged, and aliquots of plasma were frozen to $-20^{\circ} \mathrm{C}$ until the time of assay.

$m-C P P$ Ratings. Patient and control subjects completed a nine-item VAS of mood and somatic symptoms (sadness, mood swings, anxiety, irritability, global feelings, poor appetite, low energy, physical discomfort, and low self-esteem) at baseline and at 30-minute intervals for 210 minutes after oral administration of m-CPP. As described, a composite mood symptom (irritability, mood swings, sadness, and anxiety) score was calculated.

\section{Plasma Hormone Assay}

Plasma progesterone levels were assayed by a previously described radioimmunoassay method (Abraham et al. 1971). The intraassay coefficients of variation in the low and high regions of the standard curve were $5.2 \%$ and $8.4 \%$, respectively. The interassay coefficients of variation in these same regions were $13.9 \%$ and $14.3 \%$, respectively.

\section{Statistical Analysis}

\section{Fluoxetine Trial}

SYMPTOM SELF-RATINGS. The weekly means of the symptom self-ratings of both the daily VAS and the DRF during the 7 days before menses and the 7 days after the cessation of menses were compared by repeatedmeasures analysis of variance (ANOVA-R) with drug condition (baseline, placebo, and fluoxetine), cycle number (cycle 1 to 3 ), and phase (luteal and follicular) as the within-subjects variables. If no effect of number of cycle on the symptom ratings was found from the ANOVA-R analysis, the weekly means of the symptom ratings were averaged for the three cycles in each drug condition and compared using ANOVA-R, with drug condition and menstrual cycle phase as the within-subjects variables. Because of the large number of symptoms 
measured, we analyzed and present only those symptoms from the VAS and DRF most commonly endorsed by women with PMDD (i.e., the DRF symptoms listed in Table 1 and the VAS symptoms constituting the composite symptoms described).

The cross-sectional rating scales (BDI, STAI, PMTSSelf, and PMTS-Rater) were analyzed in a similar fashion to the daily self-ratings. However, cross-sectional ratings were not obtained during the baseline phase of the study. Significant differences identified by ANOVA-R were analyzed by post hoc Bonferroni $t$-tests.

We reanalyzed the data in an identical manner as that described, except that the ratings for menstrual cycles were excluded from analysis if all luteal (within 11 days prior to menses) plasma progesterone levels were less than $6.0 \mathrm{nmol} / \mathrm{l}$, suggesting an anovulatory menstrual cycle.

RESPONDER CRITERIA. For the categorical comparisons of pretreatment variables with treatment responses, responders to fluoxetine were operationally defined in three separate ways: (1) absence of a $30 \%$ increase or more (relative to the range of the VAS scale employed during baseline) in negative mood symptom score (VAS) in the luteal phase compared with the follicular phase (i.e., did not meet diagnostic criteria for PMDD during fluoxetine treatment); (2) a $50 \%$ or greater reduction of the mean of the three luteal phase weekly means in DRF composite symptom score (composite mood and social impairment scores) during fluoxetine treatment compared with baseline; and (3) results as in (2) except that only a $30 \%$ decrease in severity was required. Any subject meeting any of these criteria during the placebo phase was defined as a placebo responder.

CORRELATES Of Response to Fluoxetine. Age, past gynecological history, past personal psychiatric history, and family history of psychiatric illness in fluoxetine responders and nonresponders were compared using the Fisher exact test.

\section{m-CPP Study}

SYMPTOM SELF-RATINGS. Visual analogue symptom self-ratings during the $\mathrm{m}$-CPP challenge were analyzed by ANOVA-R, with diagnostic group (PMDD vs. controls) as the between-subjects variable and menstrual cycle phase and time (baseline, 30, 60, 90, 120, 150, 180, 210 minutes) as the within-subjects variables. Significant differences in symptom self-ratings were analyzed with post hoc Bonferroni $t$-tests. Only results from four symptoms typical of PMDD are presented.

RESPONSE CRITERIA. A negative or positive symptom response to oral $\mathrm{m}$-CPP was operationally defined as a greater than $30 \%$ increase or decrease, respectively, of the mean of four VAS mood symptom scores (sadness, anxiety, irritability, and mood lability) during the 3.5 hours of the test compared with the baseline scores.
We attempted to eliminate potential floor or ceiling effects of the patient's baseline mood symptoms in this analysis, as positive effects of $\mathrm{m}$-CPP can be obscured if the patient is asymptomatic (i.e., in the follicular phase) during testing and negative or dysphoric effects would be obscured if the patient is maximally symptomatic (i.e., in the luteal phase). Therefore, we selected the follicular phase (asymptomatic) m-CPP study to investigate negative symptom responses and the luteal phase (symptomatic) $\mathrm{m}$-CPP study to investigate positive symptom responses. Differences in the number of positive and negative symptom responses to $\mathrm{m}-\mathrm{CPP}$ between fluoxetine responders and nonresponders were compared by Fisher exact test. In addition, the symptomatic response to $\mathrm{m}-\mathrm{CPP}$ (post-m-CPP minus baseline) seen during the luteal phase was correlated (Pearson correlation coefficients) with the response to fluoxetine (fluoxetine luteal phase scores minus baseline luteal phase scores) for a composite of four VAS mood symptoms.

\section{RESULTS}

\section{Fluoxetine Trial}

Subject Characteristics. Nineteen women with PMDD were enrolled into the study, and seventeen completed the entire trial. Two women dropped out of the trial while receiving fluoxetine: one experienced intolerable migraine headaches, and the other developed irregular menstrual bleeding that was determined to be unrelated to fluoxetine administration. Participants ranged in age from 28 to 44 years (mean $\pm \mathrm{SD}, 36.5 \pm 5.4$ ); the reported duration of PMDD was $5.1 \pm 3.2$ years.

Structured diagnostic interview identified eight women $(47 \%)$ who met criteria for a past history of affective disorder [bipolar II disorder $(n=1)$ and depressive disor$\operatorname{der}(n=7)$, including postpartum depression $(n=3)]$. Four of the eight women also reported receiving treatment for these affective episodes, including lithium and antidepressants. Three women $(18 \%)$ had past histories of alcohol or substance abuse [alcohol and marijuana ( $n$ $=1)$, alcohol $(n=1)$, and cocaine $(n=1)]$. Two women reported a past history of migraine headaches. One woman had a history of hypothyroidism treated with thyroid replacement and had normal thyroid function indices prior to and during the fluoxetine trial.

Symptom ratings. No significant effects of the cycle number or of the interaction of cycle number with drug condition and menstrual cycle phase were observed in the comparisons of the weekly means of the premenstrual and postmenstrual DRF and VAS ratings for any symptom. Similarly, no significant main or interaction effects for cycle number were identified for the composite symptom scores and the cross-sectional ratings (ANOVA-R, 
$F_{2,32}<2.60, p=$ NS). Therefore, the averages of the three premenstrual and three postmenstrual weekly means of the symptom ratings were calculated during each drug condition and compared by ANOVA-R.

Daily ratings. ANOVA- $\mathrm{R}$ showed significant effects of drug condition $\left(F_{2,32}=8.2-19.4, p<.01\right)$, menstrual cycle phase $\left(F_{1,16}=6.3-109.1, p<.05\right)$, and drug condition by menstrual cycle phase interactions $\left(F_{2,32}=6.3-\right.$ $33.2, p<.01$ ) on the weekly mean of the DRF ratings of mood, physical, behavioral, and social impairment variables (Table 1), reflecting significant reductions in the mean premenstrual symptom rating scores during fluoxetine compared with baseline and placebo conditions. Specifically, post hoc testing with Bonferroni $t$-tests disclosed significantly lower anxiety, depression, irritability, mood lability, work inefficiency, social isolation, impulsiveness, food cravings, and bloating scores in subjects taking fluoxetine than in those on placebo (Bonferroni $t_{32}=3.8-6.7, p<.01$ ) and during baseline $\left(t_{32}=4.1-7.5, p<.01\right)$ (Table 1). There were no differences in mean premenstrual symptom scores for these symptoms during placebo versus baseline conditions (Bonferroni $t_{32}=0.53-2.19, p>.1$ ). In contrast, ANOVA-R did not show a significant effect of drug condition in the weekly mean symptom scores for breast pain, insomnia, or fatigue $\left(F_{2,32}=2.9,1.1\right.$, and 1.2, $p=.07, .3, .3$, respectively). However, significant effects were observed in the symptom scores for breast pain, insomnia, and fatigue for drug condition-by-menstrualcycle-phase interactions $\left(F_{2,32}=11.7,11.3\right.$, and 8 , respectively, $p<.002)$ and for menstrual cycle phase $\left(F_{1,16}=\right.$ $17.0,11.4$, and 42.2 , respectively, $p<.005$ ). Post hoc testing showed a significant decrease premenstrually in symptom scores of fatigue, insomnia, and breast pain for fluoxetine, but not for placebo, versus baseline (Bonferroni $t_{32}=3.5,3.6$, and 4.1 , respectively, $p<.01$ ). Only the symptom of decreased sexual interest did not significantly improve during either the placebo or fluoxetine trial. There were no significant differences between follicular and luteal phase rating scores in any symptoms examined during fluoxetine treatment, except for mood swings, food cravings, and bloating, suggesting some residual cyclicity in these symptoms during fluoxetine treatment (Table 1).

Composite Ratings. ANOVA- $\mathrm{R}$ showed significant effects of drug condition $\left(F_{2,32}=12.2, p<.001\right)$, menstrual cycle phase $\left(F_{1,16}=65, p<.001\right)$, and drug-byphase interactions $\left(F_{2,32}=28.3, p<.001\right)$ on the VAS composite mood rating score, reflecting a significant decrease in the premenstrual composite mood symptom scores during fluoxetine treatment versus both baseline and placebo conditions (Bonferroni $t_{32}=7.2$ and 5.87, respectively, $p<.01$ ) (Figure 1 ). No significant differ-

Table 1. Comparison of Daily Rating Form Scores for Three Treatment Conditions in Premenstrual Dysphoria

\begin{tabular}{|c|c|c|c|c|c|}
\hline \multirow[b]{2}{*}{ Symptoms } & \multirow{2}{*}{$\begin{array}{c}\begin{array}{c}\text { Baseline } \\
\text { (Mean } \pm \text { SD) }\end{array} \\
\text { Luteal }\end{array}$} & \multirow{2}{*}{$\begin{array}{c}\begin{array}{c}\text { Placebo } \\
(\text { Mean } \pm \text { SD })\end{array} \\
\text { Luteal }\end{array}$} & \multirow{2}{*}{$\begin{array}{c}\begin{array}{c}\text { Fluoxetine } \\
(\text { Mean } \pm \text { SD) }\end{array} \\
\text { Luteal }\end{array}$} & \multicolumn{2}{|c|}{$\begin{array}{c}\text { ANOVA-R } \\
\text { (F value) }\end{array}$} \\
\hline & & & & Drug & $\begin{array}{c}\text { Drug by } \\
\text { Phase }\end{array}$ \\
\hline Anxiety & $3.2(1.3)$ & $2.8(1.1)$ & $1.7(0.8)^{*,+}$ & $12.9^{* *}$ & $20.6^{* *}$ \\
\hline Depression & $3.2(0.9)$ & $2.8(1.1)$ & $1.7(0.7)^{*++}$ & $11.4^{* *}$ & $13.7^{* *}$ \\
\hline Irritability & $3.5(1.1)$ & $3.3(1.0)$ & $1.9(0.8)^{*++}$ & $17.9^{* *}$ & $17.9^{* *}$ \\
\hline Mood swings & $3.5(0.9)$ & $3.1(1.0)$ & $1.9(0.9)^{*,+}$ & $18.2^{* *}$ & $14.7^{* *}$ \\
\hline Social isolation & $2.9(1.0)$ & $2.5(1.0)$ & $1.8(0.9)^{*,+}$ & $6.5^{* *}$ & $9.1^{* *}$ \\
\hline Work inefficiency & $3.0(1.2)$ & $2.7(1.2)$ & $1.9(0.9)^{*++}$ & $5.6^{* *}$ & $16.6^{* *}$ \\
\hline Impulsiveness & $1.9(1.1)$ & $1.8(1.1)$ & $1.1(0.2)^{*++}$ & $6.4^{* *}$ & $6.9^{* *}$ \\
\hline Food cravings & $3.2(1.4)$ & $2.9(1.2)$ & $2.3(0.9)^{*++}$ & $8.2^{* *}$ & $4.8^{* *}$ \\
\hline Bloating & $3.0(1.3)$ & $3.1(1.3)$ & $2.1(1.0)^{*,+}$ & $19.1^{* *}$ & $8.2^{* *}$ \\
\hline Breast pain & $2.0(1.0)$ & $2.3(1.0)$ & $1.5(0.5)^{*+t}$ & $2.9^{+}$ & $11.7^{* *}$ \\
\hline Fatigue & $3.4(1.2)$ & $3.1(1.1)$ & $2.6(0.7)^{*}$ & $1.2^{\dagger}$ & $8.0^{* *}$ \\
\hline Insomnia & $2.6(1.1)$ & $2.2(1.3)$ & $1.9(1.2)^{*}$ & $1.1^{+}$ & $11.3^{* *}$ \\
\hline $\begin{array}{l}\text { Decreased sexual } \\
\text { interest }\end{array}$ & $3.5(1.3)$ & $3.5(1.4)$ & $3.3(1.6)$ & $1.0^{+}$ & $2.1^{\dagger}$ \\
\hline
\end{tabular}

[ANOVA-R $(\mathrm{df}=2,32)]{ }^{* *} p<.01$ for all $F$ values, except ${ }^{\dagger}$ nonsignificant.

${ }^{*} p<.01$ for fluoxetine versus baseline, (Luteal phase score) (Bonferroni $t$ comparisons).

$-p<.01$ for fluoxetine versus placebo, (Luteal phase score) (Bonferroni $t$ comparisons).

Follicular phase score (data not shown): Fluoxetine versus placebo, $p<.05$ for mood swings only.

Follicular (data not shown) versus luteal-Baseline: $p<.05$ for breast pain and decreased sexual interest; all other symptoms, $p<.01$. Placebo: insignificant for decreased sexual interest; $p<.05$ for impulsiveness and insomnia; all other symptoms $p<.01$. Fluoxetine: $p<.01$ for bloating and food cravings; $p<.05$ for mood swings; all other symptoms nonsignificant. 
ences were observed between premenstrual composite mood scores during placebo versus baseline.

Similarly, significant differences were observed in the composite social impairment score (ANOVA-R, $F_{2,32}=$ 6.9 and 14.7 for drug condition and drug-by-menstrualcycle-phase interactions, respectively, $p<.01 ; F_{1,16}=$ 37.9 for menstrual cycle phase, $p<.001$; Bonferroni $t_{32}=$ 5.5 and 4.9 for fluoxetine vs. baseline and placebo, respectively, $p<.01$; Figure 2). Composite symptom scores increased during the luteal phase from the follicular phase by $71 \%$ and $42 \%$ for baseline and placebo conditions, respectively, and by only $6 \%$ during fluoxetine treatment, further confirming a reduction of symptom cyclicity on a group basis with fluoxetine.

Bimonthly Cross-Sectional Ratings. ANOVA-R showed significant effects of drug $\left(F_{1,16}=4.6-11.3, p<.05\right)$, menstrual cycle phase $\left(F_{1,16}=27.7-105.2, p<.01\right)$, and drug-by-menstrual-cycle-phase interactions $\left(F_{1,16}=7.11\right.$ $12.3, p<.05$ ) on the averaged (over three cycles) bimonthly ratings of the BDI, STAI, PMTS-S, and PMTS-R scale scores (Figure 3 ). Post hoc testing showed significant improvements of these ratings during the late luteal phase of the menstrual cycle on fluoxetine versus placebo (Bonferroni $t_{16}=3-4.8, p<.05$ ). Moreover, post hoc testing showed no significant differences between the premenstrual and postmenstrual BDI and STAI scale scores on fluoxetine, although differences in the

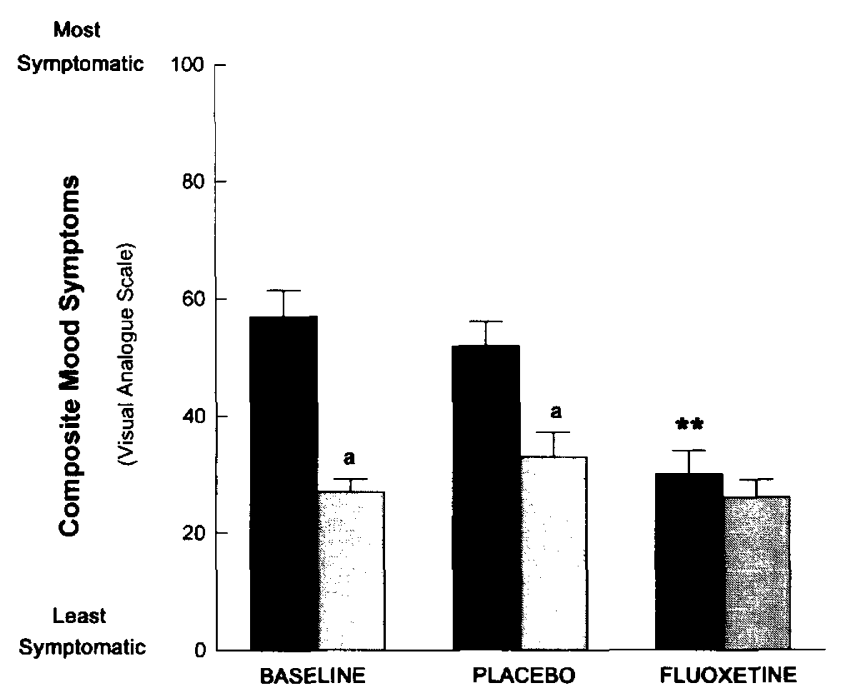

Figure 1. Fluoxetine treatment resulted in a significant reduction in the weekly means of the daily VAS composite mood symptom scores compared with both baseline and placebo conditions (Bonferroni $t$ tests ${ }^{*} p<.01$ ). No significant difference in symptom ratings was observed between baseline and placebo conditions. Significant differences between premenstrual and postmenstrual symptom scores were observed during both baseline and placebo conditions (Bonferroni $t$ tests ${ }^{a} p<.01$ ), but not during treatment with fluoxetine. Solid bars, luteal phase; gray bars, follicular phase.
PMTS-S and PMTS-R were observed. Significant differences were observed between premenstrual and postmenstrual BDI, STAI, PMTS-S, and PMTS-R during the placebo condition.

Additional Analyses. Evidence of anovulation was found in approximately $7 \%$ of menstrual cycles, reflecting occasional anovulatory cycles in six of the seventeen study participants. There were no differences in the number of anovulatory cycles during fluoxetine $(n=$ 4) compared with placebo $(n=3)$. A reanalysis of selected symptom rating scores (daily mood and social impairment ratings and cross-sectional bimonthly ratings) with the exclusion of ratings obtained during anovulatory cycles produced identical results to those observed in the original analyses.

To assess the possible effect of randomization order on symptom ratings (DRF and VAS) in the study, ANOVA-R was performed using order of drug presentation as the between-group variable, with drug condition and menstrual cycle phase as within-group variables. There were no significant effects of randomization order $\left(F_{1,16}=\right.$ $0.03-0.1, p=$ NS) or interaction effects between randomization order and drug condition and menstrual cycle phase $\left(F_{2,32}=0.15-0.59, p=N S\right)$ on the weekly mean of the composite symptom rating scores of mood and social impairment. Three out of the four women

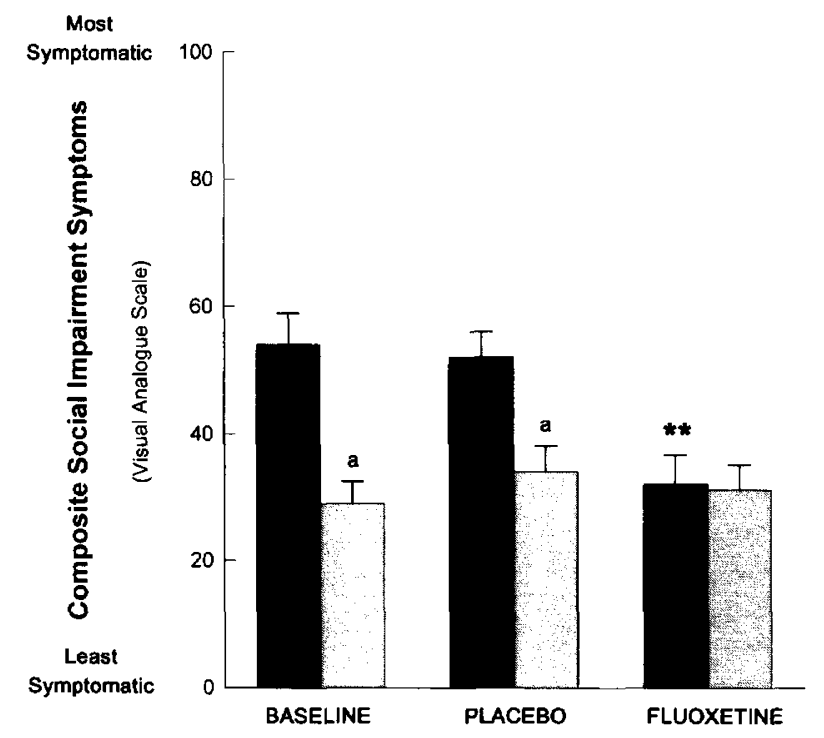

Figure 2. Fluoxetine treatment resulted in a significant reduction in the means of the daily VAS composite social impairment scores compared with both baseline and placebo conditions (Bonferroni $t$ tests ${ }^{* *} p<.01$ ). No significant difference was observed in symptom ratings between baseline and placebo conditions. Significant differences between premenstrual and postmenstrual ratings of social impairment were observed during both baseline and placebo conditions (Bonferroni $t$ tests ${ }^{a} p<.01$ ), but not during treatment with fluoxetine. Solid bars, luteal phase; gray bars, follicular phase. 
randomized to receive fluoxetine during the first 3 months and who responded to fluoxetine experienced a return of PMDD symptoms (PMTS-S rating scores in the symptomatic range) during the washout month after fluoxetine.

Dosage of Fluoxetine. The mean \pm SD daily dosage of fluoxetine during the third month of treatment was 5 , $29.9 \pm 10.6 \mathrm{mg}$, and the mean numbers of capsules (fluoxetine $10 \mathrm{mg}$ or placebo/capsule) were $2.8 \pm 0.6$ and $3.1 \pm 0.7$, respectively, for fluoxetine trial and placebo). The number of tablets of fluoxetine was not significantly different from the daily dosage of placebo during the third month (Bonferroni $t_{16}=1.3, p=\mathrm{NS}$ ).

Adverse Effects. Few side effects were reported during placebo except for fatigue $(n=2)$ and sexual dysfunction $(n=1)$. Decreased libido and/or anorgasmia, reported by six women, developed during the second and third months of fluoxetine. Five women reported symptoms of migraine-like headaches, slight confusion, and dysphoria for several days to 2 weeks after discontinuation of fluoxetine. Both sexual dysfunction and

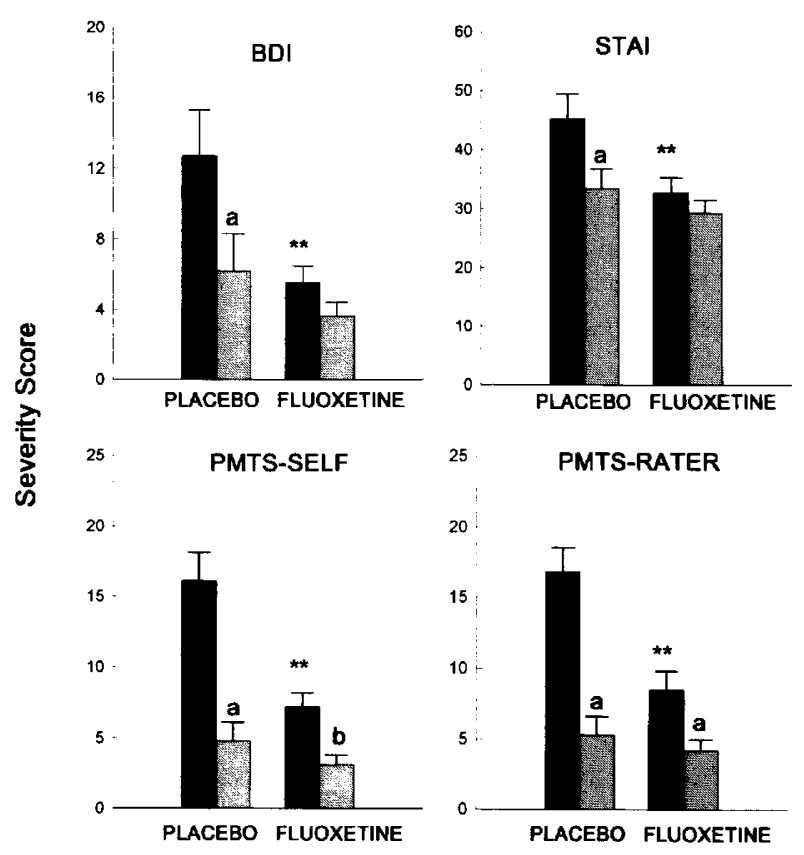

Figure 3. Significant improvements in the mean of the premenstrual ratings of the Beck Depression Inventory (BDI), Premenstrual Tension Syndrome (PMTS-Self and PMTSRater), and Spielberger State Trait Anxiety Inventory (STAI) were observed during fluoxetine treatment compared with placebo (Bonferroni $t$ tests $* *<.01$ ). Significant differences between premenstrual and postmenstrual ratings were observed in each rating scale score during placebo conditions (Bonferroni $t$ tests a $p<.01$ ) and in the PMTS ratings (Self and Rater) during fluoxetine (Bonferroni $t$ tests ${ }^{a} p<$ $\left..01,{ }^{\mathrm{b}} p<.05\right)$. Solid bars, luteal phase; gray bars, follicular phase. symptoms during withdrawal appeared more frequently during fluoxetine than during placebo (Fisher exact test, $p=.08$ and $p<.05$, respectively).

Correlates of Response to Fluoxetine. Eleven of seventeen women who completed the trial $(65 \%)$ were identified as responders on the basis of failure to meet criteria for PMDD during fluoxetine treatment with absence of response to placebo. Ten women (59\%) demonstrated a $30 \%$ or greater reduction in symptom scores, and seven women $(41 \%)$ demonstrated a $50 \%$ reduction in symptom scores relative to baseline during fluoxetine, but not placebo conditions. Only three women showing a remission of mood cyclicity (no longer met PMDD criteria) did not have a $30 \%$ improvement in PMDD symptoms. Thus the $30 \%$ criterion failed to identify three of eleven women who met the cyclicity criterion; conversely, the cyclicity criterion failed to identify two of ten women who met the $30 \%$ criterion. One woman who had more than a $50 \%$ reduction in symptoms still experienced mood cyclicity. Employing the same three sets of response criteria (i.e., syndromal response, $50 \%$, and $30 \%$ improvement over baseline) resulted in $n=3,2$, and 4 women, respectively, being identified as placebo responders. All women responding to placebo also were responders to fluoxetine but were not included as responders in subsequent comparisons.

No differences in the mean fluoxetine dosage between responders and nonresponders were found, irrespective of the criteria used (50\% symptom reduction: $29.5 \pm 5.2$ vs. $26.7 \pm 6.5 \mathrm{mg} /$ day; $30 \%$ symptom reduction: $27.3 \pm 5.8$ vs. $28.6 \pm 6.6 \mathrm{mg} /$ day). There were no significant differences between fluoxetine responders and nonresponders in age, age of onset of illness, pregnancy history, past history of alcohol or drug abuse, or family history of mental illness. Using a 50\% symptom reduction criterion for fluoxetine responders, $7(70 \%)$ of 10 nonresponders compared with $1(14 \%)$ of 7 responders had a positive past psychiatric history (Fisher exact test, $p=.05$ ). In contrast, using a $30 \%$ symptom reduction criterion for fluoxetine responders, 4 of 7 nonresponders compared with 4 of 10 responders had a positive past psychiatric history (Fisher exact test, $p=\mathrm{NS}$ ).

\section{m-CPP Test}

Twelve patients and ten controls received oral m-CPP challenge tests. Nine patients and all controls had tests during both the follicular and luteal phases. The remaining three patients had only one test [follicular $(n=$ $2)$, luteal $(n=1)]$, as they experienced distressing side effects related to the first $\mathrm{m}$-CPP challenge.

ANOVA-R demonstrated significant effects of diagnosis, menstrual cycle phase, time (trend), and the interaction of diagnosis, phase, and time on the symptom 
self-ratings of sadness $\left(F_{7,126}=2.2-10.9, p<.05\right)$, reflecting the improvement of sadness following $\mathrm{m}-\mathrm{CPP}$ administration in PMDD patients, but not controls, during the luteal phase. Post hoc testing showed that the improvement from baseline was maximal at 120 and 180 minutes post-m-CPP (Bonferroni $t_{126}=3.3-4.9, p<.01$ ) and was maintained until approximately 210 minutes. Similar effects were observed for symptoms of mood swings, irritability, anxiety, global feeling, low energy, self-esteem, physical discomfort, and composite symptoms, although significance was not reached for all effects measured in each symptom. In contrast, ANOVA-R showed no significant diagnostic effects in appetite.

No significant correlation was observed between the maximal change in premenstrual mood symptom scores during the luteal phase $\mathrm{m}-\mathrm{CPP}$ and the reduction of those symptoms (from baseline) during fluoxetine treatment $(r=0.2, p=\mathrm{NS})$. Of the women who did not respond to fluoxetine, equal numbers $(n=3)$ showed a positive response or an absence of a positive response to $\mathrm{m}-\mathrm{CPP}$.

\section{DISCUSSION}

We observed a significant improvement in mood and behavioral and physical symptoms during fluoxetine treatment under double-blind controlled conditions in women with prospectively confirmed diagnoses of PMDD. Our response rate was between $41 \%$ and $65 \%$ of the women in the sample depending on the criteria we employed. On a group basis, the positive response to fluoxetine in the women with PMDD in this study was similar to response patterns observed in previous trials of fluoxetine (Stone et al. 1991; Wood et al. 1992; Menkes et al. 1993; Steiner et al. 1995) as well as in controlled trials of other serotonergic agents, including fenfluramine (Brzezinski et al. 1990), buspirone (Rickels et al. 1989), and clomipramine (Sundblad et al. 1992, 1993), and open-label trials of fluoxetine (Brandenburg et al. 1993), the $5-\mathrm{HT}_{2}$ antagonist and 5- $\mathrm{HT}$ reuptake inhibitor nefazodone (Freeman et al. 1994), and L-tryptophan (Steinberg et al. 1994). In contrast, one previous study (Veeninga et al. 1990) in women with self-reported diagnoses of PMS that were not confirmed longitudinally prior to study entry failed to observe a significant improvement in premenstrual symptoms with fluvoxamine compared with placebo. The results of Veeninga et al. (1990) may reflect differences in entry criteria (subjects may not have met severity criteria employed in other studies) or, alternatively, may suggest a different mechanism of action and, therefore, a different pattern of response in women with PMS (and possibly PMDD) to fluvoxamine compared with other serotonergic agents (Brosen 1993; Caccia et al. 1993; Mustafa and Alhaider 1993; Carroll 1994; Sacchetti et al. 1994).
We observed syndromal remission (as defined by the failure to meet diagnostic criteria for PMDD during fluoxetine treatment but not placebo) in $65 \%$ of the sample compared to complete or full remission rates (using different criteria from those employed in this study) of approximately $50 \%$ in two previous controlled trials of fluoxetine in women with PMS (Stone et al. 1991; Menkes et al. 1992, 1993). Although our findings are similar to those seen in previous trials, the slightly enhanced frequency of response observed in our study may reflect our use of a variable dose regimen of fluoxetine compared to the fixed $20 \mathrm{mg}$ daily dose of fluoxetine employed in these other studies. However, on a group basis, we did not identify a significant effect of dose on symptom response to fluoxetine, consistent with the report by Steiner et al. (1995).

Alternatively, some variation in the report of response rates across the studies may be due to differences in the criteria employed to define therapeutic response. For example, Stone et al. (1991) reported 50\% of the sample studied no longer met criteria for LLPDD, but we observed a rate of $65 \%$ using similar criteria. Moreover, Stone et al. (1991) identified an additional four women who experienced a greater than $50 \%$ reduction in the mean premenstrual symptom scores on fluoxetine compared to baseline, suggesting that response rates are criteria-dependent. However, different criteria appear to identify somewhat different (not just smaller or larger) groups as responders. In fact, when we employed a $50 \%$ reduction criterion based on the difference between premenstrual symptom scores during fluoxetine compared to baseline, our observed response rate declined to $41 \%$, considerably lower than the response rates using syndrome-based criteria. In contrast, Wood et al. (1992) reported a response rate to fluoxetine in women with PMS of approximately $87 \%$ (seven of eight subjects) based on the observed reduction in symptoms on fluoxetine compared to baseline. These results reflected the improvement in seven of eight subjects, but the degree of improvement or the extent of residual cyclicity in individual subjects was not described. Similarly, in a recent, double-blind, placebocontrolled, parallel design study (Steiner et al. 1995) response rates of $55 \%$ and $28 \%$ to fluoxetine and placebo, respectively, were observed when a $50 \%$ symptom reduction criterion was employed. Interpretation of response requires the ability to distinguish between symptom improvement, remission of symptom cyclicity, and syndromal remission. Despite the fact that we observed symptom improvement and elimination of symptom cyclicity (significant follicular to luteal phase differences) for the majority of individual PMDD target symptoms, significant differences remained between phases in the scores on the PMTS-S and PMTS-R scales, suggesting the persistence on a group basis of some symptom cyclicity. These differences in the pattern and degree of 
symptomatic response to fluoxetine may simply reflect the diversity of premenstrual symptoms and the differential sensitivity of certain symptoms to fluoxetine. Some trials of serotonergic agents (Stone et al. 1991; Daaman et al. 1992; Sundblad et al. 1992; Wood et al. 1992), but not all (Menkes et al. 1992, 1993; Freeman et al. 1994), have reported a greater response in emotional and behavioral symptoms than in the physical symptoms of PMS. In fact, Sundblad et al. (1993) have suggested that the differential response rates of physical symptoms, such as bloating and breast pain, compared to emotional symptoms, such as irritability, may reflect a different pathophysiological basis for these types of symptoms. We observed an improvement in both breast pain and bloating in addition to emotional symptoms, consistent with reports by both Menkes et al. (1993) and Freeman et al. (1994) and suggesting a similar basis of response to fluoxetine for both physical and emotional symptoms. However, we did observe residual symptom cyclicity in bloating as well as in mood swings and food cravings [similar to observations by Stone et al. (1991)]. These symptoms may represent residual premenstrual molimina (Magyar et al. 1979) that is no longer amplified by the premenstrual affective state. Nevertheless, it is possible that the observed differences in the pattern and degree of symptom response to fluoxetine will predict the subsequent long-term response.

The effects of fluoxetine in this sample of women were not due to fluoxetine-induced alterations of reproductive endocrine function. All women reported normal menstrual cycle function during the trial, and there was no increased frequency of anovulatory cycles during fluoxetine compared with placebo, similar to the report by Wood et al. (1992), but in contrast to Menkes et al. (1993), who observed that three of sixteen women experienced a change in menstrual cycle function during fluoxetine treatment and none during placebo.

Symptom cyclicity, defined as a difference in follicular and luteal phase composite symptom scores, decreased from $72 \%$ to $42 \%$ on placebo and to $6 \%$ on fluoxetine, reflecting modest, nonstatistically significant improvement during the placebo phase presumably related to nonspecific factors, including clinic visits and participation in the study. Three women in the sample met response criteria during the placebo phase (as well as during the fluoxetine phase) representing a somewhat higher number of placebo responders in this group than in the study by Wood et al. (1992). However, the response rate to placebo $(18 \%)$ in our patients is similar to that found in the reports by Stone et al. (1991), Menkes et al. (1992, 1993), and Steiner et al. (1995) (19\% to $28 \%)$.

Response rates to fluoxetine in women with PMS/ PMDD are similar to those described in depression (Stark and Hardison 1985; Feighner et al. 1991; Bowden et al.
1993), panic disorder (Gorman et al. 1987; Schneier et al. 1990), and obsessive-compulsive disorder (Pigott et al. 1990; Tollefson et al. 1994). However, in contrast to the onset of action of fluoxetine in other psychiatric conditions (6-8 weeks), we observed a rapid onset of response to fluoxetine, usually during the first month of treatment, similar to the time course reported by Steiner et al. (1995) and Menkes et al. (1993), who observed that seven of 16 women improved on fluoxetine after the first 2 weeks of treatment. Moreover, the beneficial effects on the symptoms of PMDD of acute serotonin stimulation with $\mathrm{m}$-CPP that we observed and the reports of the efficacy of the luteal phase administration of clomipramine (Sundblad et al. 1993) in women with PMS suggest that the mechanism of action of these agents in PMS/PMDD is not the classical antidepressant or antiobsessive effect. Furthermore, the return of typical PMDD symptoms soon after discontinuation of fluoxetine in this and other studies (Stone et al. 1991; Menkes et al. 1993; Pearlstein and Stone 1994) suggests that treatment of PMDD (even for up to 10 months) does not produce the sustained remission seen more frequently in the treatment of major depressive illness.

Wood et al. (1992) reported that one woman in the trial did not respond to fluoxetine and was later identified as having a past history of depression. The authors suggested that a past history of depression may predispose to a nonresponse to fluoxetine, at least at the 20$\mathrm{mg}$ daily dose. Further support for this suggestion was provided by Pearlstein and Stone (1994), who reported that a past history of major depression was associated with a partial response to fluoxetine under open-labeled variable-dose $(20-40 \mathrm{mg})$ maintenance conditions. We observed no difference in the past psychiatric history between responders and nonresponders to fluoxetine when a $30 \%$ reduction in symptom severity from baseline criterion was employed. However, as did Wood et al. (1992) and Pearlstein and Stone (1994), we observed a significant increase in the frequency of a past history of major depression in the nonresponders compared with the responders to fluoxetine when the more stringent $50 \%$ symptom improvement criterion was employed, suggesting that a previous major depression may in fact complicate the response of PMDD symptoms to fluoxetine. If confirmed in subsequent trials, the differential pattern of PMDD response to fluoxetine may be the first clinical characteristic in women with PMDD distinguishing those who have from those who have no past history of major depression. In contrast, although self-reported age of onset of PMDD may not be reliably recalled, we could not confirm observations by Pearlstein and Stone (1994) of an earlier age of onset of PMDD predicting a poor response to fluoxetine, since we did not observe any difference in the retrospective self-reports of the age of onset of PMDD between responders and nonresponders to fluoxetine. 
Seven of ten women receiving $\mathrm{m}$-CPP during the luteal phase experienced an acute improvement in their symptoms, and all seven later responded to fluoxetine, although two of these women also responded to placebo. The three women whose symptoms did not improve with $\mathrm{m}$-CPP did not meet the $50 \%$ improvement criterion during fluoxetine; therefore, the nonresponse to $\mathrm{m}-\mathrm{CPP}$ may be an additional predictor of nonresponse to fluoxetine. Interestingly, two of these three women not responding to either $\mathrm{m}-\mathrm{CPP}$ or fluoxetine had past histories of affective disorders. A larger sample size would be necessary to confirm or disconfirm the value of these predictors.

In general, fluoxetine was well tolerated by the women in this study. The emergence of adverse effects was minimal, even when the employed dose range was increased to approximately 30 to $40 \mathrm{mg}$ in the third month. However, in contrast to other researchers, we observed an increased frequency of reports of disturbed libido and anorgasmia during fluoxetine compared to baseline and placebo conditions. Thirty-five percent of the women reported decreased libido and/or anorgasmia, an incidence similar to that found in other conditions treated with fluoxetine (Herman et al. 1990; Jacobsen 1992), but somewhat higher than the $17 \%$ reported by Pearlstein and Stone (1994). An association between suicidal ideation and fluoxetine use (Teicher et al. 1990) was not evident in this sample. However, ratings of the impulse to hurt self or others were diminished by fluoxetine, as similarly reported by Pearlstein and Stone (1994).

In conclusion, this study confirms several previous trials reporting the effectiveness of fluoxetine and other serotonergic agents in treating women with PMDD. Both serotonergic agents and ovarian suppression have been consistently reported to improve symptoms in the majority of women with PMS, and these two categories of treatments may act via different mechanisms (i.e., elimination of cyclic gonadal hormone secretion vs. serotonergic system stimulation or downregulation) as suggested by Wood et al. (1992). In addition to the shared efficacy of these treatments in PMS, neither ovarian suppression nor serotonergic agents are effective in all women with otherwise identical clinical presentations. Further investigations of the possible historical antecedents, such as past history of major depression, or biological variables, such as nonresponse to 5- $\mathrm{HT}$ challenge, may help in identifying a pattern of factors predictive of response to these individual treatments.

\section{ACKNOWLEDGMENTS}

This paper was presented at the 48th Annual Meeting of the Society of Biological Psychiatry in San Francisco on May 22, 1993. The authors wish to thank Eli Lilly Company at Indianapolis, Indiana, for providing fluoxetine hydrochloride.

\section{REFERENCES}

Abraham GE, Swerdloff R, Tulchinsky D, Odell WD (1971): Radioimmunoassay of plasma progesterone. J Clin Endocrinol Metab 32:619-624

American Psychiatric Association (1994): Diagnostic and Statistical Manual of Mental Disorders, ed 4. Washington, DC, American Psychiatric Press

Bancroft J, Cook A, Davidson D, Bennie J, Goodwin G (1991): Blunting of neuroendocrine responses to infusion of L-tryptophan in women with perimenstrual mood change. Psychol Med 21:305-312

Beck AT, Ward CH, Mendelson M, Mock J, Erbaugh J (1961): An inventory for measuring depression. Arch Gen Psychiatr 4:561-571

Bowden CL, Schatzberg AF, Rosenbaum A, Contreras SA, Samson JA, Dessain E, Sayler M (1993): Fluoxetine and desipramine in major depressive disorder, J Clin Psychopharmacol 13:305-311

Brandenbrug S, Tuynman-Qua H, Verheij R, Pepplinkhuizen L (1993): Treatment of premenstrual syndrome with fluoxetine: An open study. Int Clin Psychopharmacol $8: 313-317$

Brosen K (1993): The pharmacogenetics of the selective serotonin reuptake inhibitors. Clin Invest 71:1002-1009

Brzezinski AA, Wurtman JJ, Wurtman RJ, Gleason R, Greenfield J, Nader T (1990): d-Fenfluramine suppresses the increased calorie and carbohydrate intakes and improves the mood of women with premenstrual depression. Obstet Gynecol 76:296-301

Caccia S, Anelli M, Codegoni AM, Fracasso C, Garattini S (1993): The effects of single and repeated anorectic doses of 5-hydroxytryptamine uptake inhibitors on indole levels in rat brain. Br J Pharmacol 110:355-359

Carroll BJ (1994): Are SSRI antidepressants clinically homogeneous? Lancet 344:550

Daaman MJ, Brown WA (1992): Single-dose fluoxetine in management of premenstrual syndrome. J Clin Psychiatr 53:210-211

Endicott J, Halbreich U (1982): Retrospective report of premenstrual depressive changes: Factors affecting confirmation by daily ratings. Psychopharmacol Bull 18:109112

Eriksson E, Lisjo P, Sundblad C, Andersson K, Andersch B, Modigh K (1990): Effect of clomipramine on premenstrual syndrome. Acta Psychiatr Scand 81:87-88

Feighner JP, Gardner EA, Johnston JA, Batey SR, Khayrallah MA, Ascher JA, Lineberry CG (1991): Double-blind comparison of bupropion and fluoxetine in depressed outpatients. J Clin Psychiatr 52:329-335

Freeman EW, Rickels K, Sondheimer SJ, Denis A, Pfeifer S, Weil S (1994): Nefazodone in the treatment of premenstrual syndrome: A preliminary study. J Clin Psychopharmacol 14:180-186

Gorman JM, Liebowitz MR, Fyer AJ, Goetz D, Campeas RB, Eyer MR, Davies SO, Klein DF (1987): An open trial of fluoxetine in the treatment of panic attacks. J Clin Psychopharmacol 7:329-332

Halbreich U (1990): Gonadal hormones and antihormones, serotonin and mood. Psychopharmacol Bull 26:291-295 
Halbreich U, Endicott J, Lesser J (1985): The clinical diagnosis and classification of premenstrual changes. Can J Psychiatr 30:489-497

Harrison WM, Endicott J, Nee J (1990): Treatment of premenstrual dysphoria with alprazolam: A controlled study. Arch Gen Psychiatr 47:270-275

Herman JB, Brotman AW, Pollack MH (1990): Fluoxetineinduced sexual dysfunction. J Clin Psychiatr 51:25-27

Hollander E, Stein DJ, DeCaria CM, Saoud JB, Klein DF, Liebowitz MR (1993): A pilot study of biological predictors of treatment outcome in obsessive-compulsive disorder. Biol Psychiatr 33:747-749

Jacobsen FM (1992): Fluoxetine-induced sexual dysfunction and an open trial of yohimbine. J Clin Psychiatr 53:119122

Magyar DM, Boyers SP, Marshall JR, Abraham GE (1979): Regular menstrual cycles and premenstrual molimina as indicators of ovulation. Obstet Gynecol 53:411-414

Menkes DB, Taghavi E, Mason PA, Spears GFS, Howard RC (1992): Fluoxetine treatment of severe premenstrual syndrome. Br Med J 305:346-347

Menkes DB, Taghavi E, Mason PA, Howard RC (1993): Fluoxetine's spectrum of action in premenstrual syndrome. Int Clin Psychopharmacol 8:95-102

Murphy DL, Mueller EW, Hill JL, Tolliver TJ, Jacobsen FM (1989): Comparative anxiogenic, neuroendocrine, and other physiologic effects of m-chlorophenylpiperazine given intravenously or orally to healthy volunteers. Psychopharmacology 98:275-282

Mustafa AA, Alhaider AA (1993): Differential effects of fluoxetine on isoprenaline-stimulated water intake in ethanol-treatment rats: A role for beta-adrenoceptors. Eur J Pharmacol 237:147-154

Pearlstein TB, Stone AB (1994): Long-term fluoxetine treatment of late luteal phase dysphoric disorder. J Clin Psychiatr 55:332-335

Pigott TA, Pato MT, Bernstein SE, Grover GN, Hill JL, Tolliver, TJ, Murphy DL (1990): Controlled comparisons of clomipramine and fluoxetine in the treatment of obsessive-compulsive disorder: Behavioral and biological results. Arch Gen Psychiatr 47:926-932

Rickels K, Freeman E, Sondheimer S (1989): Buspirone in treatment of premenstrual syndrome. Lancet 1:777

Sacchetti E, Conte G, Guarneri L (1994): Are SSRI antidepressants a clinically homogeneous class of compounds? Lancet 344:126-127

Schmidt PJ, Grover GN, Rubinow DR (1993): Alprazolam in the treatment of premenstrual syndrome: A doubleblind, placebo-controlled trial. Arch Gen Psychiatr 50:467-473

Schneier FR, Liebowitz MR, Davies SO, Fairbanks J, Hollander E, Campeas R, Klein DF (1990): Fluoxetine in panic disorder. J Clin Psychopharmacol 10:119-121

Smith S, Rinehart JS, Ruddock VE, Schiff I (1987): Treatment of premenstrual syndrome with alprazolam: Results of a double-blind, placebo-controlled, randomized crossover clinical trial. Obstet Gynecol 70:37-43

Spielberger CB, Gorsuch RL, Luschene RE (1970): STAI manual for the state-trait anxiety inventory. Palo Alto, CA, Consulting Psychologists Press

Spitzer RL, Williams JB, Gibbon M, First MB (1989): Structured Clinical Interview for DSM-III-R, Patient Edition. New York, Biometrics Research Department, New York State Psychiatric Institute

Spoont MR (1992): Modulatory role of serotonin in neural information processing: Implications for human psychopathology. Psychol Bull 112:330-350

Stark P, Hardison CD (1985): A review of multicenter controlled studies of fluoxetine versus imipramine and placebo in outpatients with major depressive disorder. J Clin Psychiatr 46:53-58

Steinberg S, Annable L, Young SN, Beanger M (1994): Tryptophan in the treatment of late luteal phase dysphoric disorder: A pilot study. J Psychiatr Neurosci 19:114-119

Steiner M, Haskett RF, Carroll BJ (1980): Premenstrual tension syndrome: The development of research diagnostic criteria and new rating scales. Acta Psychiatr Scand 62:177-190

Steiner M, Steinberg S, Stewart D, Carter D, Berger C, Reid R, Grover D, Streiner D (1995): Fluoxetine in the treatment of premenstrual syndrome. $\mathrm{N}$ Engl J Med 332: 1529-1534

Stone AB, Pearlstein TB, Brown WA (1991): Fluoxetine in the treatment of late luteal phase dysphoric disorder. J Clin Psychiatr 52:290-293

Sundblad S, Modigh K, Andersch B, Eriksson E (1992): Clomipramine effectively reduces premenstrual irritability and dysphoria: A placebo-controlled trial. acta Psychiatr Scand 85:39-47

Sundblad C, Hedberg MA, Eriksson E (1993): Clomipramine administered during the luteal phase reduces the symptoms of premenstrual syndrome: A placebo-controlled trial. Neuropsychopharmacology 9:133-145

Teicher MH, Glod C, Cole JO (1990): Emergence of intense suicidal preoccupation during fluoxetine treatment. Am J Psychiatr 147:207-210

Tollefson GD, Rampey Jr AH, Potvin JH, Jenike MA, Rush AJ, Dominguez RA, Koran LM, Shear K, Goodman W, Genduso LA (1994): A multicenter investigation of fixed-dose fluoxetine in the treatment of obsessive-compulsive disorder. Arch Gen Psychiatr 51:559-567

VanKammen DP, Murphy DL (1975): Attenuation of the euphoriant and activating effects of d- and l-amphetamine by lithium carbonate treatment. Psychopharmacologia 44:215-224

Veeninga AT, Westenberg HGM, Weusten JTN (1990): Fluvoxamine in the treatment of menstrually related mood disorders. Psychopharmacology 102:414-416

Wood SH, Mortola JF, Chan Y, Moossazadeh F, Yen SSC (1992): Treatment of premenstrual syndrome with fluoxetine: A double-blind, placebo-controlled, crossover study. Obstet Gynecol 80:339-344 